\title{
Photoacoustic detection of phase transitions at low temperatures in $\mathrm{CsPbCl}_{3}$ crystals
}

\author{
Masanobu Iwanaga* \\ Department of Physics, Graduate School of Science, \\ Tohoku University, Sendai 980-8578, Japan
}

November 6, 2018

\begin{abstract}
Phase transitions in $\mathrm{CsPbCl}_{3}$ crystals have been explored by photoacoustic (PA) method in a wide temperature range of $150-300 \mathrm{~K}$. The PA signals, induced by ns-pulsed laser excitation of electronic states and detected with a piezoelectric transducer, are thermoelastic ultrasonic waves lasting for hundreds of microseconds. The wavelet analysis of the PA wave has clarified that the thermoelastic wave includes several $\mathrm{MHz}$ components. The PA signals have been made use of in order to examine phase transitions at low temperatures in the crystal. Changes of PA signals indicate that two phase transitions take place at low temperatures around $200 \mathrm{~K}$. The types of phase transitions are discussed taking into recent experimental results.
\end{abstract}

Keywords: Photoacoustic spectroscopy; Ultrasonic wave; Phase transitions; $\mathrm{CsPbCl}_{3}$

\footnotetext{
*E-mail: iwanaga@phys.tohoku.ac.jp
} 


\section{INTRODUCTION}

$\mathrm{CsPbCl}_{3}$ crystal was investigated as a typical material undergoing structural phase transitions above room temperature (RT) (Fuiii. Hoshino. Yamada and Shirane, 1974); the crystal is perovskite in the temperature range above $320 \mathrm{~K}$, transforms to tetragonal at $320 \mathrm{~K}$, and becomes orthorhombic or monoclinic at $310 \mathrm{~K}$. The phase transitions are considered to be ferroelastic, related to zone-boundary phonons of $\mathrm{M}_{3}$ and $\mathrm{R}_{25}$ (Fuiii et al., 1974; Hirotsu, 1971).

On the other hand phase transition below RT was explored in the measurement of doped $\mathrm{CsPbCl}_{3}$ with the electron-paramagnetic-resonance (EPR) method, so that the results implied that phase transition takes place at about 180 K (Cape. White and Feigelson, 1969; Cohen. Young and Chang, 1971). However the temperature of phase transition estimated from the EPR experiments is 185 (Cape et al., 1969) and $176 \mathrm{~K}$ (Cohen et al., 1971), and slightly different with each other. The discrepancy could come from the doping effect on the phase transition, and therefore it seems more appropriate to examine the non-doped crystal for determining the phase-transition point. The crystal structure below RT has been studied recently with the x-ray diffraction (Sakuma. Mutou. Ohki. Akai. Takahashi and Ishii, 2002); the study concluded that the crystal is orthorhombic below RT and implied that structural transition does not take place below RT. Thus phase transition below RT is not conclusive from the structural examination. The result implies that if phase transition does take place below RT, it can be secondorder transition that is not associated with any crystallographic transition.

Another recent study on phase transition at low temperatures has been performed by focusing on optical absorption tail and photoluminescence $(\mathrm{PL})$ of $\mathrm{CsPbCl}_{3}$. Since the crystal is a semiconductor with direct bandgap (Heidrich. Schäfer. Schreiber. Söchtig. Grandke and Stolz, 1981), the electronic excited states are generated under photoexcitation. The absorption tail and PL properties suddenly change around $170 \mathrm{~K}$ Havashi. Kobavashi. Iwanaga and Watanabe, 
2001); the results suggest phase transition below RT.

To explore the phase transition, photoacoustic spectroscopy (PAS) is a suitable method because PAS enables us to observe self-induced thermoelastic ultrasonic wave under photoexcitation. In fact phase transitions have been often studied by PAS and detected successfully (Jackson and Amer, 1980; Etxebarria. Uriarte. Fernández. Tello and Gómez-Cuevas, 1984); even second-order transition were observed. In the present study, it is aimed to clarify the properties of observed PA signals and furthermore to test the existence of phase transition below RT.

Experimental details are described in Sec. 2 Experimental results are presented and the wavelet analysis is applied to the PA wave in Sec. 3. The wavelet analysis has the advantage of resolving the superimposed waves into the several waves corresponding to each frequency component; for example, the electric noise mixed in the experimental data is apparently separated from PA signals. After clarifying PA-signal properties, the existence of phase transitions below RT is discussed in Sec. 4.

\section{EXPERIMENTAL DETAILS}

$\mathrm{CsPbCl}_{3}$ crystals were grown with the Bridgman technique. To purify 99.999\% $\mathrm{PbCl}_{2}$ powder, the single crystal was previously grown, and $99.99 \%$ $\mathrm{CsCl}$ powder and the $\mathrm{PbCl}_{2}$ single crystal were mixed at equimolar amount and sealed in a quartz ampoule at about $10^{-5}$ Torr. The ampoule was set in a Bridgman furnace and was moved from the point of $970 \mathrm{~K}$ to that of $720 \mathrm{~K}$ at about $20 \mathrm{~K}$ /day. Though the melting temperature of $\mathrm{CsPbCl}_{3}$ is about $880 \mathrm{~K}$, the ampoule was kept below the melting point for several days to anneal the specimen. Finally it was cooled from $720 \mathrm{~K}$ to RT for a day. The as-grown ingot of $\mathrm{CsPbCl}_{3}$ was aggregate of single crystals.

The crystal was cleaved in $a b$ plane perpendicular to $c$ axis; the crystals can be cleaved only in $a b$ plane. Typical dimension of the cleaved specimen 
was $5 \times 5 \times 1 \mathrm{~mm}^{3}$ along $a, b$ and $c$ axes respectively. Both cleaved $a b$ planes were used for PA measurement; one was the plane injected by incident photons and the other was firmly attached to a piezoelectric transducer (PZT) with conductive organic paste. Experimental configuration is schematically drawn in Fig. 1]

Two type of PZT were used; the PZT of 3.99-MHz resonant frequency was a plate of $5 \times 5 \times 1 \mathrm{~mm}^{3}$ and that of $1.97-\mathrm{MHz}$ resonant frequency was a 10-mm diameter and 1-mm thickness disc. One of the cleaved planes was attached to a PZT as shown in Fig. 1. Curie temperatures of the PZTs were $603 \mathrm{~K}$ and therefore the PZTs do not undergo any phase transition in the temperature range of $150-300 \mathrm{~K}$ at which the present measurement was performed. The crystal and PZT were set in a He-flow cryostat. Acousticwave ringing effect in a PZT was checked; the effect is compared with the PA wave propagated from $\mathrm{CsPbCl}_{3}$ in the next section.

The PA signals were induced by the third harmonic generation of Nd:YAG laser; the laser pulses were $3.49 \mathrm{eV}$ and 5 -ns width, and the reputation was $10 \mathrm{~Hz}$. Since $\mathrm{CsPbCl}_{3}$ crystals have the fundamental absorption edge at $3.0 \mathrm{eV}$ (Heidrich et al., 1981), the incident light is absorbed due to the electronic interband transition, except for the surface reflection. The absorption takes place in the thin surface layer because of the absorption length less than $1 \mu \mathrm{m}$ and the sample thickness of $1 \mathrm{~mm}$. In this configuration the PA signal generated in the surface layer propagates through the crystal and is detected by the PZT on the back surface. Thus the detected PA wave is bulk wave propagated through the crystal; the measured PA wave does not only propagate along $c$ axis but also includes the off-axis wave which travels along $a$ - or $b$-axis and the multiple reflection in the crystal. The intensity of incident laser light was about $200 \mu \mathrm{J} /$ pulse, and the incident light is loosely focused at the spot size of 1-mm diameter on the sample surface; the sample was not damaged by the laser-light irradiation. The PA wave was measured by an oscilloscope without any preamplifier. Different time-range data were 
measured by varying the coupling resistance to the oscilloscope.

The amplitude and phase of the PA signals were picked up by a twophase lockin amplifier at the cycle of incident pulse. The reference input to the lockin detector was taken from the transistor-transistor-logic pulses synchronized with the laser pulses. In the setup the lockin detector pick up the signals of the same frequency with the reference; the amplitude is proportional to the peak intensity because the lockin detector automatically tunes the phase of sine curve to the peak position of PA signal. Measuring the PA signals by the lockin, the coupling resistance is required high; the PA wave is therefore broadened to ms-order, so that the lockin technique is applicable to the present measurement.

At the end the relation of the pulsed PA measurement with the conventional PA measurement is made mention of; pulsed PA signals are generally stronger than the conventional PA signals induced by chopper-modulated

laser light because the laser pulses have higher photon density (Patel and Tam, 1981). In the conventional PA measurement using cw laser light of $3.81 \mathrm{eV}$ and a chopper, the amplitude and phase of $\mathrm{PA}$ signals of $\mathrm{CsPCl}_{3}$ show a profile similar to those of pulsed PA signals (see Fig. (4). The PA signals were just weaker than the pulsed PA signals. Consequently there is not any special advantage to employ the conventional PA technique here.

\section{RESULTS AND ANALYSIS}

Figure 2 shows detected PA wave at $296 \mathrm{~K}$ (curve (a)) in a $\mu$ s range. The wave was induced by $3.49-\mathrm{eV}$ pulsed laser light of 5 -ns width at $t=12.5 \mu \mathrm{s}$. The PA wave continues to oscillate prominently for more than $80 \mu \mathrm{s}$. For comparison with the PA wave, PZT ringing (curve (b)) was also displayed to a similar scale; it was measured under direct photoirradiation onto the PZT attached to nothing but lead wires. The ringing in the PZT is directly stimulated due to the laser pulse, independent of photon energy of laser 
pulses. The envelope of rapid component decays exponentially at about 10 $\mu$ s and the slow component weakly survives up to $100 \mu$ s. As seen in Fig. 2 the decay of PA wave is different from the PZT ringing. That is, the ringing effect on the detected PA wave is not dominant. Probably the oscillatory structure of PA wave mainly comes from long lasting multiple reflection of the thermoelastic wave in the crystal. Besides the PZT ringing such as curve (b) in Fig. 2 becomes comparable to the PA waves only under direct and 1.5-times more intense excitation. Thus, though the influence of ringing is in principal included in the measured PA wave, the contribution is quite small. It is also shown in the analysis of Fig. 3.

Figure 3(a) presents the PA wave at $297 \mathrm{~K}$, which was detected by a PZT of resonant frequency of $1.97 \mathrm{MHz}$. Solid line in Fig. 3(a) represents the measured PA wave, and dashed line shows the incident laser pulse of $3.49 \mathrm{eV}$.

To analyse this real time oscillation, we choose to use the wavelet method. In the present analysis, the fourth B-spline wavelet $\psi_{4}$ in the inset of Fig. 3(b) is selected from many wavelets; the $\psi_{4}$ is a finite wave packet composed of third-order polynomials and corresponds mainly to a narrow frequency band via Fourier transformation (Sakakibara, 1994). First the measured PA wave in Fig. 3(a) is approximated by the fourth B spline. A good approximation is displayed as $f_{0}(t)$ in Fig. 3(b). The PA wave $f_{0}(t)$ is decomposed as follows: $f_{0}(t)=\Sigma_{j} g_{j}(t)$ where $g_{j}(t)=\Sigma_{k} d_{k}^{(j)} \psi_{4}\left(2^{j} t-k\right)$, and $d_{k}^{(j)}$ are coefficients (Sakakibara, 1994). The $f_{0}$ is well resolved by $g_{j}(j=-1, \cdots,-6)$. Figure 3(b) displays the result of wavelet decomposition. $g_{-1}$ and $g_{-2}$ make small contribution; the $g_{-2}$ represents electric noise at $25 \mu$ s which appears without PA signal and stems from the trigger signal for measurement. $g_{-4}$ is apparently predominant, and $g_{-3}$ and $g_{-5}$ contribute to some extent. Though $g_{-6}$ also contributes slightly, it almost corresponds to the PZT ringing observed in this time range. $g_{-3}, g_{-4}$ and $g_{-5}$ are consequently regarded as the contributors responsible for the PA wave. $g_{-4}$ corresponds to the 
frequency band at $1.5 \mathrm{MHz}$. On the other hand $g_{-6}$ does to the band at 0.4 $\mathrm{MHz}$; the frequency is distinctive from the PZT resonant frequency of 1.97 $\mathrm{MHz}$, that is, the ringing just stems from multiple reflection of acoustic wave in the PZT. Thus, by removing the electric noise and ringing components, the PA component in time domain is found to be superposition of a few frequency bands.

At the end of PA-wave analysis, it is to be noted that PA wave is almost invariant in 150-300 K. Therefore PA wave at $296 \mathrm{~K}$ was analysed here.

PA-signal amplitude (solid circle) and phase (dot) are plotted against temperature (150-250 K) in Fig. 4. They were measured, by using $10-\mathrm{Hz}$ and 3.49-eV laser pulses and a PZT of resonant frequency of $1.97 \mathrm{MHz}$, with the raising and lowering rates of $1.2 \mathrm{~K} / \mathrm{min}$. The incident light was parallel to $c$ axis. The amplitude and phase measured with decreasing temperature indicate a prominent peak and dip at $198 \mathrm{~K}$ respectively while those with increasing temperature have a similar peak and dip at $231 \mathrm{~K}$. Another prominent change in the phase, that is, the leap by two degrees is observed at $177 \mathrm{~K}$ in the data on cooling. Arrows indicate the lower edge of phase change. The origins of changes are discussed in Sec. 4.

Tens of jumped data points appear for both amplitude and phase only in the data on cooling. The irregular data presumably originate from unexpected cracking of the crystal on cooling because the irregularity is far less on heating. Besides it appears very differently on second cooling or in another sample. However the prominent dips and peaks are reproduced; it is therefore relevant that they indicate intrinsic changes in the crystal.

Concerning with the cracking, there are a few remarks: (i) The PA measurement was begun at RT and kept to $150 \mathrm{~K}$. Before measuring PA signals on heating, the sample was cooled to about $120 \mathrm{~K}$. It was due to the thermal controller. (ii) The excess cooling probably induces further cracking. In fact, the amplitude of PA signals on heating is about half on cooling (see Fig. (4). (iii) In the present setup of Fig. 1 PA wave travels through the 
crystal. The cracking consequently decreases measured PA signals.

\section{DISCUSSION ON PHASE TRANSITIONS}

The changes of PA-signal amplitude and phase in Fig. 4 is discussed in this section. As shown in Figs. 22 and 3, PA wave consists of thermoelatic ultrasonic components. Therefore the amplitude and phase detected by a two-phase lockin amplifier correspond to those of thermoelatic wave. The amplitude is proportional to the peak intensity in two-phase lockin equipment. The phase generally depends on the amplitude. Figure 4 shows the amplitude (closed circles) and phase (dots) of thermoelastic wave vs temperature. The intensity has tens of microvolts at 150-250 K while the phase increases as temperature becomes lower. The gradual changes come from the thermal deformation of crystal.

The changes at 198 and $231 \mathrm{~K}$ in Fig. 4 are probably due to those of propagation of bulk thermoelastic wave; the change seems different from gradual thermal deformation of the crystal and from the change of the PZT which has Curie point at $603 \mathrm{~K}$. The changes are therefore ascribed to a phase transition at the temperatures. Indeed they show similar profiles except for irregular data. The transition has large hysteresis between cooling and heating.

A theoretical study on photoacoustic detection of phase transition (Etxebarria et al., 1984) showed that the profiles of amplitude and phase of PA signals are similar to those at a second-order phase transition. It is therefore likely that the transition at 198 and $231 \mathrm{~K}$ is second-order. Arrows in Fig. [4 indicate the lower edge of phase and correspond to the transition points estimated from

the indication by Etxebarria et al. (1984). According to the calculation, the amplitude decreases to zero at a first-order transition because of ultrasonic attenuation of structural transition.

As noted in Sec. 1 phase transitions below $\mathrm{RT}$ in $\mathrm{CsPbl}_{3}$ have been 
hard to be detected. This is because structural study by x-ray diffraction is ineffective in the crystal (Sakuma et al., 2002). The result is consistent with the assignment that the type of transition at 198 and $231 \mathrm{~K}$ is secondorder. In addition another phenomenological analysis for second-order phase transition (Landau and Lifshitz, 1984) derives the leap of sound velocity and the increase of absorption coefficient of ultrasonic wave at second-order phase-transition point when lattice distortion couples with the square of order parameter; it is often called Landau-Khalatnikov mechanism. The dips of amplitude in Fig. 4 are explained by the increase in absorption coefficient of $\mathrm{MHz}$-thermoelastic wave in the crystal.

On the other hand another possibility for the type of transition still exists. The study employing x-ray diffraction indicated that the crystal, keeping the crystallographic group, varies the length of unit cell along $a$ and $c$ axes while it hardly changes the length along $b$ axis (Sakuma et al., 2002). It is thus possible that slight displacement of lattice takes place in the unit cell; the slight change might not be well discriminated in the x-ray-diffraction study. Recently order-disorder transition was reported at $193 \mathrm{~K}$ by Raman scattering (Carabatos-Nédelec. Oussaid and Nitsch, 2003); the assignment is consistent with the possibility of slight structural change. Consequently there are two possibilities at present; the type of transition at 198 and 231 $\mathrm{K}$ is due to second-order or the slight structural transition in the unit cell.

The leap of phase at $177 \mathrm{~K}$ in Fig. 4 appears on cooling and is sharper than the change at $198 \mathrm{~K}$. It is improbable that the sudden change at $177 \mathrm{~K}$ comes from the adiabatic deformation of the crystal. That is, it also suggests the existence of a phase transition at the temperature. The transition is most likely different from that at $198 \mathrm{~K}$ because the profiles are inconsistent with each other. The leap at $177 \mathrm{~K}$ is possibly due to a second-order phase transition though the type is not conclusive just like that at $198 \mathrm{~K}$.

The leap at $177 \mathrm{~K}$ is observed only on cooling. The reason is presumably as follows: as remarked at the end of Sec. 3, the amplitude of PA signal 
on heating is two-times weaker than that on cooling, because of cracking. It is consequently harder to detect the slight change of PA signal on heating. Indeed the leap at $177 \mathrm{~K}$ on cooling is the same order with the noisy fluctuation of signals. Thus the leap on heating may be hidden.

The cracking perhaps connects to the ferroelastic nature at low temperatures. Ferroelastic domains are typically several micrometers in various materials. The boundary of domains prevents PA wave from propagating for long distance up to $1 \mathrm{~mm}$. On the other hand PL properties mainly depend on the local structure within several tens of nanometers. Indeed PL shows hysteresis on cooling and heating. The explanation for the absence of PA-signal leap on heating is compatible with the change of PL properties.

In conclusion, $\mathrm{PA}$ signals in $\mathrm{CsPCl}_{3}$ crystals have been studied below RT; it has been shown that the leap of the PA signals appears at 177 and 198 $\mathrm{K}$ on cooling and at $231 \mathrm{~K}$ on heating. Though the profiles imply secondorder phase transition, the slight structural transition in the unit cell is not excluded. Thus the types are not yet conclusive at present. However phase transitions below RT are confirmed by examining PA signals. The transition at $177 \mathrm{~K}$ is probably responsible for the leap of EPR signals (Cohen et al., 1971) and for the sudden change of optical and PL properties (Havashi et al., 2001).

\section{Acknowledgments}

I would like to acknowledge Dr. K. Yoshino (Miyazaki Univ.) for introducing me to PAS, and also thank Mr. T. Kobayashi for the growth of $\mathrm{CsPbCl}_{3}$ crystals and Prof. T. Hayashi (Kyoto Univ.) for support to the study. This

work was partly supported by Grant-in-Aid for Research Fellow of the Japan Society for the Promotion of Science. 


\section{References}

Cape, J.A., White, R.L. and Feigelson, R.S. (1969). EPR study of the structure of $\mathrm{CsPbCl}_{3}$. J. Appl. Phys., 40, 5001.

Carabatos-Nédelec, C., Oussaid, M. and Nitsch, K. (2003). Raman scattering investigation of cesium plumbochloride, $\mathrm{CsPbCl}_{3}$, phase transitions. J. Raman Spectrosc., 34, 388.

Cohen, M.I., Young, K.F. and Chang, T.T. (1971). Phase transitions in $\mathrm{CsPbCl}_{3}$. J. Appl. Phys., 42, 5267.

Etxebarria, J., Uriarte, S., Fernández, J., Tello, M.J. and Gómez-Cuevas A. (1984). Piezoelectric photoacoustic detection of first- and second-order phase transitions. J. Phys. C.: Solid State Phys., 17, 6601.

Fujii, Y., Hoshino, S., Yamada, Y. and Shirane, G. (1974). Neutronscattering study on phase transitions of $\mathrm{CsPbCl}_{3}$. Phys. Rev. B, 9, 4549, and earlier references cited therein.

Hayashi, T., Kobayashi, T., Iwanaga, M. and Watanabe, M. (2001). Exciton dynamics related with phase transitions in $\mathrm{CsPbCl}_{3}$ single crystals. $J$. Lumin., 94-95, 255.

Heidrich, K., Schäfer, W., Schreiber, M., Söchtig, J., Grandke T. and Stolz, H.J. (1981). Electronic structure, photoemission spectra, and vacuumultraviolet optical spectra of $\mathrm{CsPbCl}_{3}$ and $\mathrm{CsPbBr}_{3}$. Phys. Rev. B, 24, 5642 .

Hirotsu, S. (1971). Experimental studies of structural phase transitions in $\mathrm{CsPbCl}_{3}$. J. Phys. Soc. Jpn., 31, 552.

Jackson, W. and Amer, N.M. (1980). Piezoeletric photoacoustic detection: Theory and experiment. J. Appl. Phys., 51, 3343. 
Landau, L.D. and Lifshitz, E.M. (1984). Statistics Physics. ButterworthHeinemann, Oxford.

Patel, C.K.N. and Tam, A.C. (1981). Pulsed optoacoustic spectroscopy of condensed matter. Rev. Mod. Phys., 53, 517.

Sakakibara, S. (1994). In Wavelets: Theory, Algorithms, and Applications, edited by Chui, C.K., L. Montefusco and L. Puccio. Academic Press, New York. pp. 179-196.

Sakuma, T., Mutou, M., Ohki, K., Akai, M., Takahashi, H. and Ishii, Y. (2002). Low-energy excitation in $\mathrm{CsPbX}_{3}(\mathrm{X}=\mathrm{Cl}, \mathrm{Br})$. Solid State Ionics, 154-155, 237. 


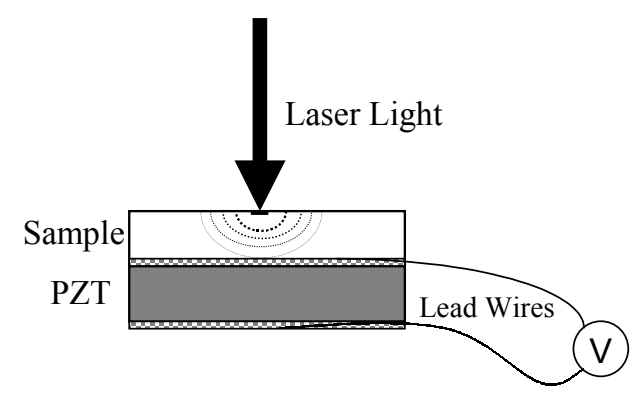

Figure 1: Schematic configuration of a $\mathrm{CsPbCl}_{3}$ and a $\mathrm{PZT}$. The sample was photoexcited on one cleaved plane and PA signals were detected on the other by the PZT attached with conductive organic paste. Dotted lines in the sample stand for the PA waves schematically. The piezoelectric voltage was measured along the thickness direction. This figure is drawn for the PZT of $5 \times 5 \times 1 \mathrm{~mm}^{3}$. More details are described in Sec. 2. 


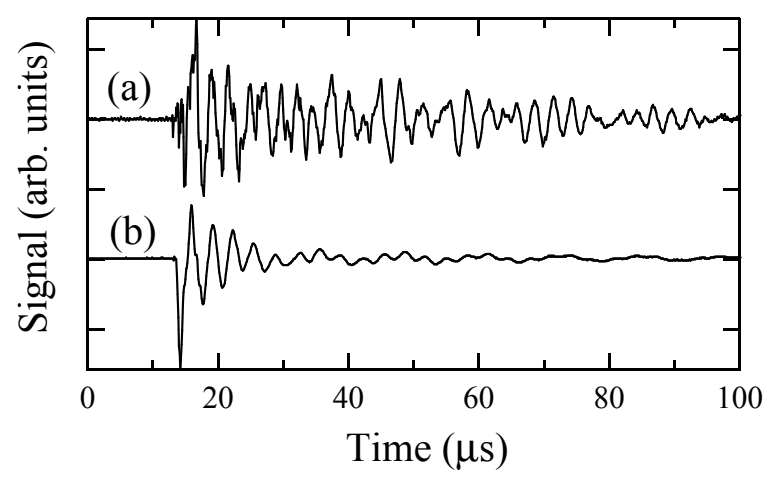

Figure 2: (a) Detected PA wave induced by photoexcitation of $\mathrm{CsPbCl}_{3}$ at $296 \mathrm{~K}$ with 3.49-eV laser pulses of 5 -ns width. Incident laser pulses reach onto the surface at $12.5 \mu \mathrm{s}$. The PZT used in the measurement has the resonance at $3.99 \mathrm{MHz}$. (b) Ringing at $296 \mathrm{~K}$ in a PZT of $3.99-\mathrm{MHz}$ resonance; it was induced by direct photoinjection onto the PZT. The incident light was 2.33-eV laser pulses of 5-ns width. 3.49-eV laser pulses also induce the same shape of ringing. The ringing effect on the detected PA wave is described in the text. Both spectra are presented to a similar scale. 

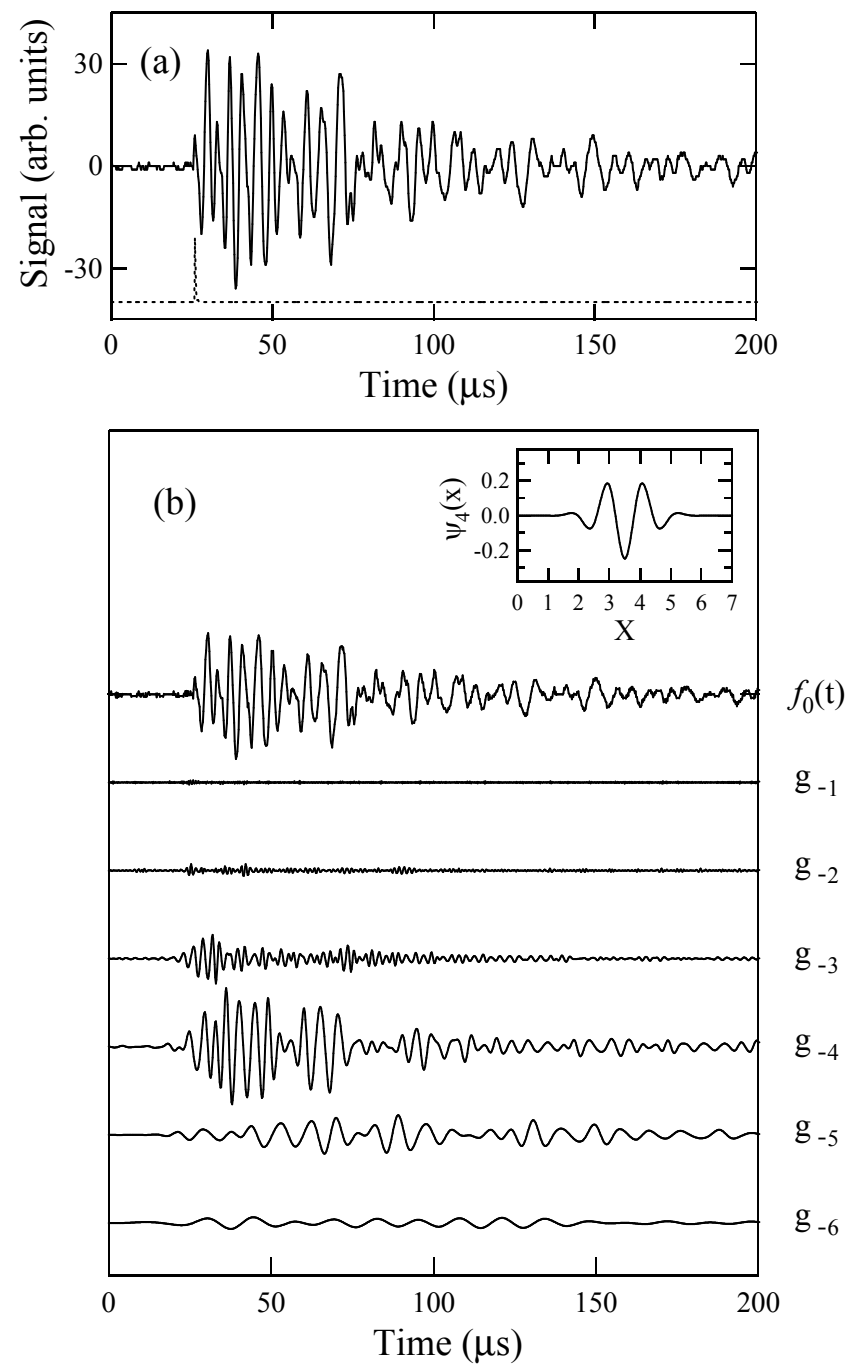

Figure 3: (a) PA wave in $\mathrm{CsPbCl}_{3}$ at $297 \mathrm{~K}$ (solid line); it is induced by 5ns-width laser pulses of $3.49 \mathrm{eV}$. Dashed line: the position at which the laser pulses reach onto the sample surface. The dashed line is vertically offset. (b) Wavelet analysis with the fourth B-spline wavelet $\psi_{4}$; the inset shows the $\psi_{4}$ which is center symmetrical. $f_{0}(t)$ denotes a good approximation for the PA wave in Fig. B(a). $g_{j}(j=-1, \cdots,-6)$ resolve $f_{0}$; they are vertically offset and displayed to the same scale with $f_{0}$. 

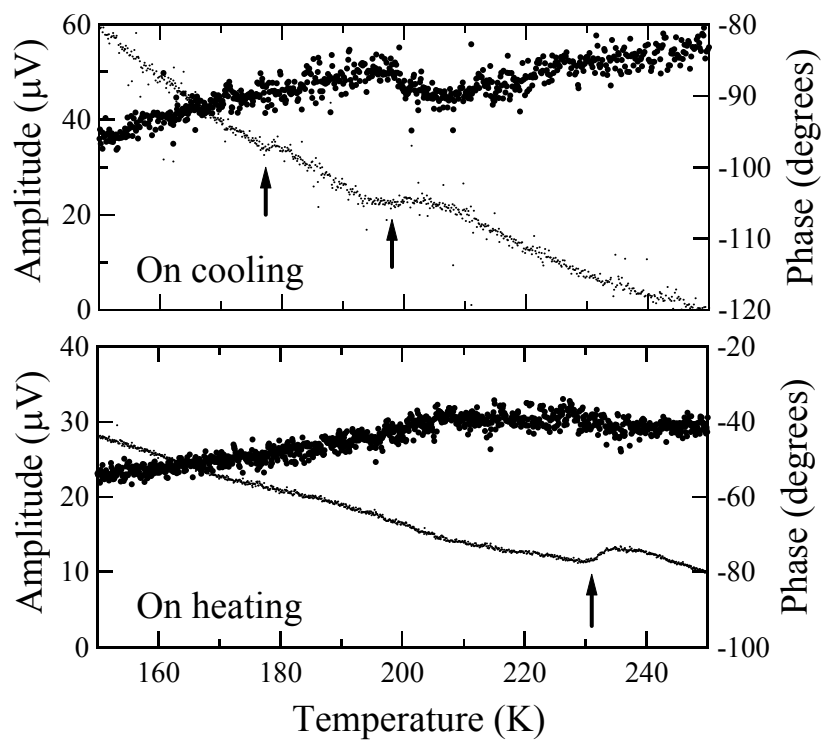

Figure 4: Amplitude (closed circle) and phase (dot) of PA signal vs temperature. The incident light was $10-\mathrm{Hz}$ and $3.49-\mathrm{eV}$ pulsed laser light and the incident direction was parallel to $c$ axis. The resonant frequency of PZT was $1.97 \mathrm{MHz}$. The increasing and decreasing rates of temperatures were 1.2 $\mathrm{K} / \mathrm{min}$. Arrows indicate the lower edge of phase change and are indicative of phase transitions; the discussion on phase transitions is given in Sec. 4 